\title{
Treatment Options and Goals for Patients with Generalized Pustular Psoriasis
}

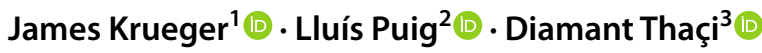

Accepted: 14 November 2021 / Published online: 21 January 2022

(c) The Author(s) 2022

\begin{abstract}
Generalized pustular psoriasis (GPP) is a rare, severe neutrophilic skin disorder characterized by sudden widespread eruption of superficial sterile pustules with or without systemic inflammation. GPP flares can be life-threatening if untreated due to potential severe complications such as cardiovascular failure and serious infections. Currently, there are no GPPspecific therapies approved in the USA or Europe. Retinoids, cyclosporine, and methotrexate are the most commonly used non-biologic therapies for GPP. The evidence that supports the currently available treatment options is mainly based on case reports and small, open-label, single-arm studies. However, recent advances in our understanding of the pathogenic mechanisms of GPP and the identification of gene mutations linked to the disease have paved the way for the development of specific targeted therapies that selectively suppress the autoinflammatory and autoimmune mechanisms induced during GPP flares. Several biologic agents that target key cytokines involved in the activation of inflammatory pathways, such as tumor necrosis factor- $\alpha$ blockers and interleukin (IL)-17, IL-23, and IL-12 inhibitors, have emerged as potential treatments for GPP, with several being approved in Japan. The evidence supporting the efficacy of these agents is mainly derived from small, uncontrolled trials. A notable recent advance is the discovery of IL36RN mutations and the central role of IL-36 receptor ligands in the pathogenesis of GPP, which has defined key therapeutic targets for the disease. Biologic agents that target the IL-36 pathway have demonstrated promising efficacy in patients with GPP, marking the beginning of a new era of targeted therapy for GPP.
\end{abstract}

Digital Features for this article can be found at https://doi.org/ 10.6084/m9.figshare.16823503.

Diamant Thaçi

diamant.thaci@uksh.de

1 Laboratory for Investigative Dermatology, The Rockefeller University, New York, NY, USA

2 Department of Dermatology, Hospital de la Santa Creu i Sant Pau, Universitat Autònoma de Barcelona, Barcelona, Spain

3 Institute and Comprehensive Center for Inflammation Medicine, University of Lübeck, Lübeck, Germany

\section{Key Points}

There are no generalized pustular psoriasis (GPP)specific therapies approved in the USA or Europe for the treatment of GPP and management of GPP flares.

The evidence supporting the use of non-biologic and biologic therapies for the treatment of patients with GPP is limited and mainly based on case studies and small, open-label, single-arm studies.

Advances in our understanding of the pathogenesis of GPP have led to the development of targeted therapies, such as interleukin-36 receptor inhibitors, which have shown promising efficacy and acceptable safety in earlyphase clinical trials. 
Graphical abstract

$\triangle$ Adis

OPEN

ACCESS

PEER-REVIEWED
INFOGRAPHIC

Treatment Options and Goals for Patients With Generalized Pustular Psoriasis

\author{
James Krueger, Lluís Puig, Diamant Thaçi
}

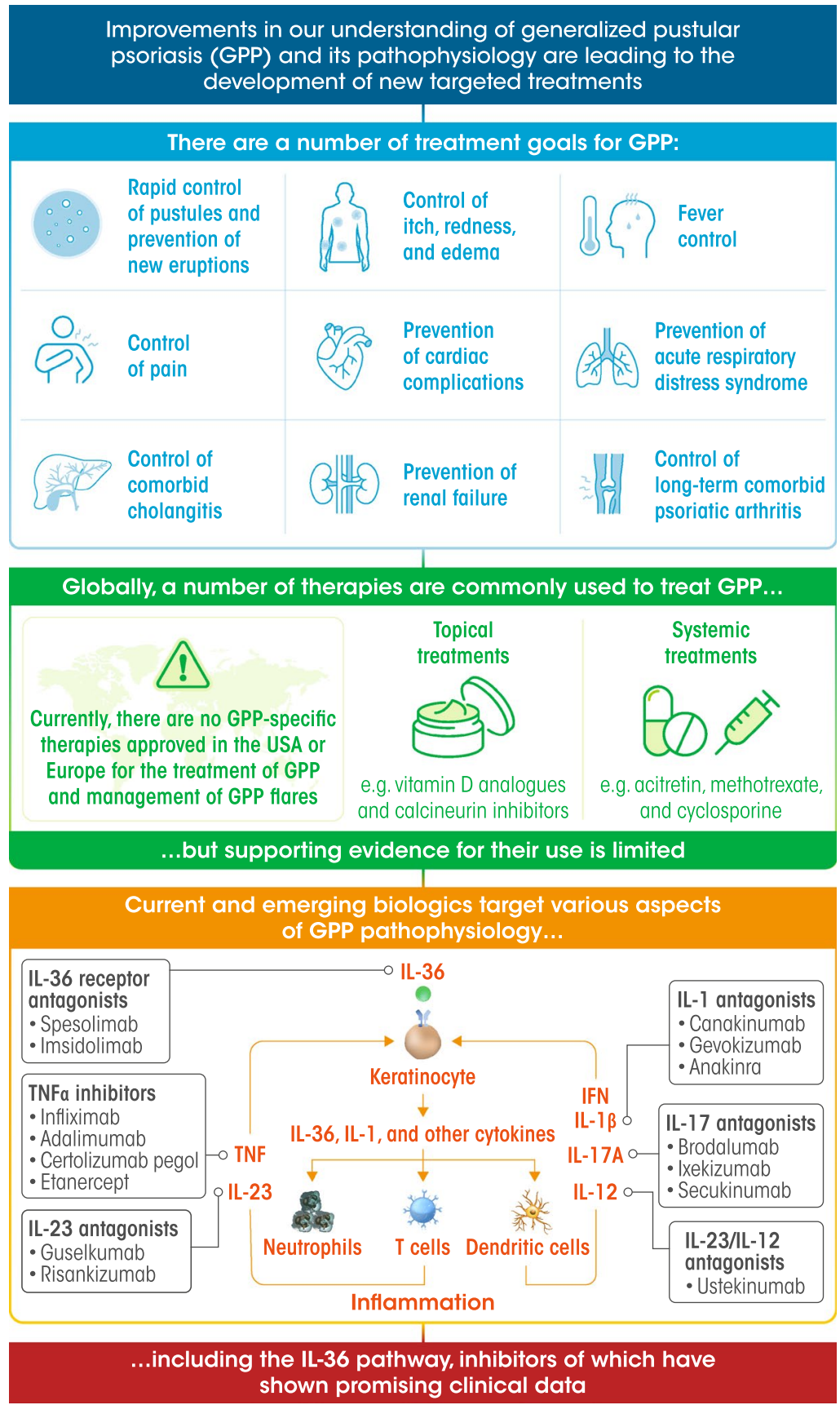

The infographic represents the opinion of the authors. For a full list of declarations, including funding and author disclosure statements, please see the full text online.

(C) The authors, CC-BY-NC 2021 


\section{Introduction}

Generalized pustular psoriasis (GPP) is a rare, severe skin disease characterized by sudden widespread eruption of macroscopically visible primary sterile pustules on nonacral skin and may or may not be associated with systemic inflammation $[1,2]$. Approximately $65 \%$ of patients diagnosed with GPP have a prior diagnosis of plaque psoriasis [3]. According to the European Rare and Severe Psoriasis Expert Network (ERASPEN), the clinical course of GPP can be relapsing, at least once, or persistent for more than 3 months [2]. GPP flares can be life-threatening if untreated due to potential systemic complications, such as bacterial infections [4], prerenal insufficiency [5], and cardiovascular failure [1]. GPP flares can be triggered by the use or discontinuation of corticosteroids [3, 6]. In addition, paradoxical incidence of GPP flares has been reported with the use of methotrexate, ustekinumab, and tumor necrosis factor (TNF)- $\alpha$ blockers $[3,7]$. Upper respiratory tract infections, pregnancy, and stress have also been identified as triggers for GPP flares [4, 6, 8].

The pathogenic mechanisms of GPP flares are poorly understood. However, recent advances in our understanding of the biology and genetic mechanisms of autoinflammation and autoimmunity have led to the characterization of critical genetic mutations associated with the incidence and pathogenesis of GPP (these are discussed in greater detail in Chapter 2 [https://doi.org/10.1007/s40257-02100655-y] of this supplement). Most notably, IL36RN mutations have been identified in cases of sporadic and familial GPP from around the world [9-14]. These loss-of-function mutations in the interleukin (IL)-36 receptor antagonist result in the hyperactivation of IL-36 signaling due to the unopposed stimulation of the IL-36 receptor by its ligands, IL-36 $\alpha$, IL36 $\beta$, and IL-36 $\gamma$. The increased production of IL-36 induces the production of chemokines by keratinocytes, leading to neutrophil epidermal accumulation, which drives the pathogenesis of GPP, and the formation of the characteristic spongiform pustules of Kogoj [3, 15, 16]. The proinflammatory functions of IL-36 cytokines can be further potentiated by a positive feedback loop with the IL-17/IL-23 axis. In addition, expression of IL-36 $\gamma$ has been found to correlate with disease activity in psoriasis and was suppressed by TNF $\alpha$ inhibition [17]. Accordingly, several potential therapeutic targets have been identified based on our understanding of these mechanisms. Inhibition of TNF $\alpha$, IL-1, and IL-17A, which stimulate IL-36 $\alpha$, IL-36 $\beta$, and IL-36 $\gamma$ synthesis in keratinocytes, may potentially disrupt inflammatory pathways in GPP (Fig. 1) [18]. Similarly, targeting the IL-23 pathway, which regulates the synthesis of IL-17, could potentially impact the IL-36 axis in patients with GPP [1]. Among these pharmacologic targets, inhibition of the
IL-36 receptor is being evaluated for the treatment of GPP flares [19].

Advances in our understanding of the genetics and pathogenesis of GPP have revealed new opportunities to develop GPP-specific targeted therapeutic strategies. In this review, we highlight the currently available treatments and the emerging treatment options for GPP flares and long-term management of GPP, and provide recommendations for treatment goals.

\section{Current Treatment Options for Patients with GPP}

There are currently no GPP-specific treatments approved in the USA or Europe [1]. All treatments discussed herein have no prescribing label for GPP except in Japan, where several biologics are currently approved for treatment of the disease, including the TNF $\alpha$-blocking agents adalimumab, infliximab, and certolizumab pegol; the IL-17/IL-17R inhibitors secukinumab, brodalumab, and ixekizumab; and the IL-23 inhibitors risankizumab and guselkumab [20-26]. Brodalumab is also approved in Taiwan and Thailand [20, 26, 27].

Current treatment options for GPP can be classified into biological and non-biological systemic agents. Based on the Japanese guidelines for the management of GPP and the Medical Board of the National Psoriasis Foundation, the most commonly used treatments for patients with GPP are retinoids, cyclosporine, and methotrexate (Table 1) $[1,20$, 28-31]. However, the evidence that supports the use of current therapies for GPP is largely ill-defined and mainly based on small, open-label, single-arm studies and case reports. Care should be taken when basing treatment decisions on data from case reports to avoid the risk of overinterpreting the findings. It is also important to note the potential for publication bias, as positive treatment results are predominantly reported and may not truly reflect the number of cases of unsuccessful treatment. With no therapies approved outside of Japan, there is a lack of up-to-date and globally relevant GPP-specific treatment guidelines and goals despite the distinct pathologic and clinical features of the disease. Therefore, treatment of GPP flares and long-term management of patients with GPP remain urgent unmet medical needs.

\subsection{Non-Biologic Systemic Therapies}

\subsubsection{Methotrexate}

Methotrexate is a widely used drug for autoinflammatory and autoimmune conditions and is recommended for use in GPP according to the 2018 Japanese guidelines and the 2012 Medical Board of the National Psoriasis Foundation guidelines [20, 


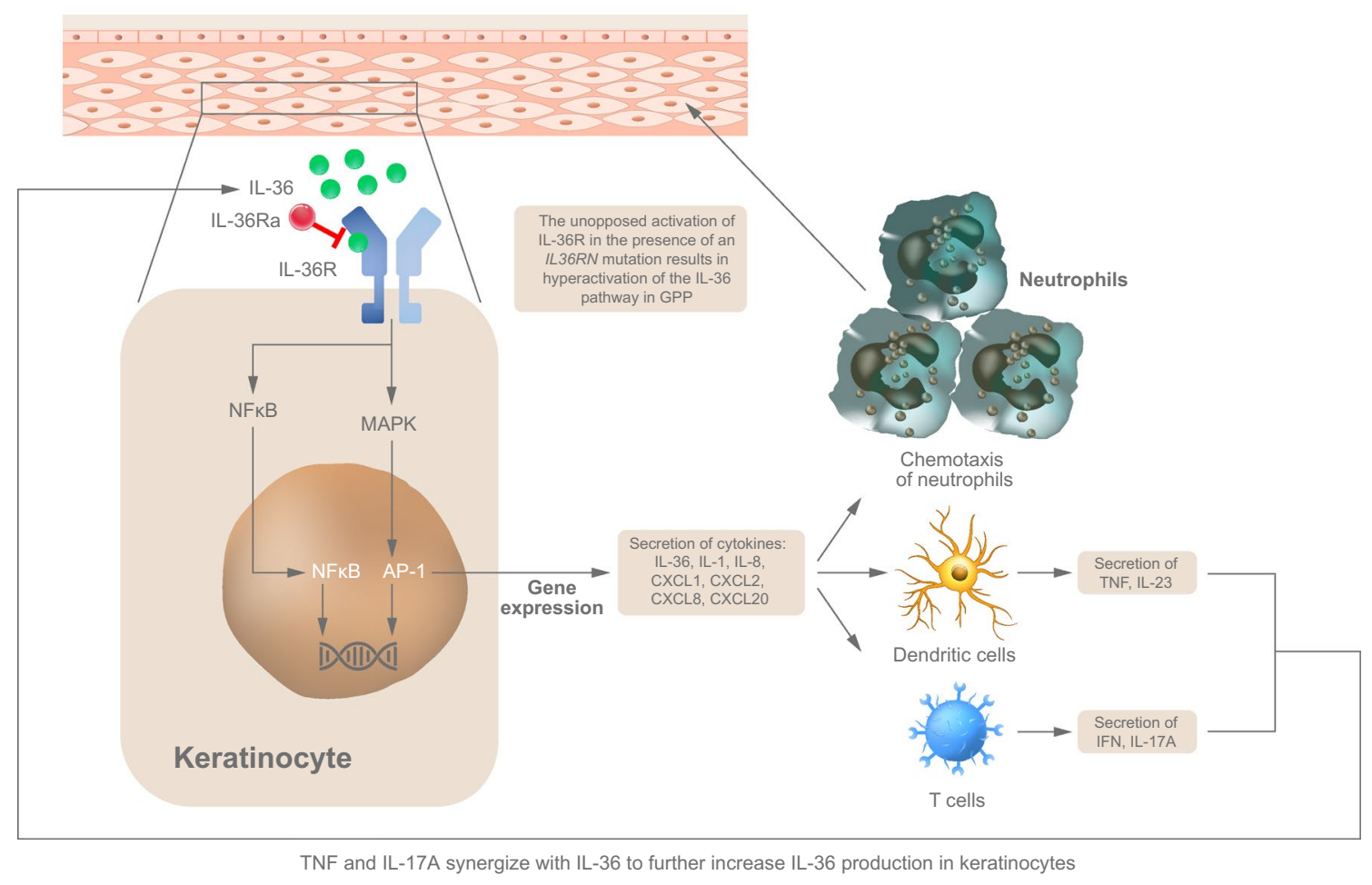

Fig. 1 Hyperactivation of IL-36 plays a central role in the pathogenesis of GPP by driving neutrophilic involvement. GPP generalized pustular psoriasis, $I F N$ interferon, $I L$ interleukin, $R$ receptor, $T N F$ tumor necrosis factor. Adapted from Zhou et al. [18]

28, 29]. However, there are no clinical studies that demonstrate the efficacy of methotrexate for the treatment or prevention of GPP flares. Although the mechanism of action of methotrexate for the treatment of GPP is not well defined, recent findings in psoriasis suggest that it restores the immunosuppressive function of regulatory $\mathrm{T}$ cells through inhibition of the mammalian target of rapamycin (mTOR) pathway [29, 32].

In patients with psoriasis, methotrexate has a slow onset of action. In a retrospective study that included 157 patients with several types of psoriasis, including 12 with GPP, methotrexate resulted in skin clearance in $31 \%$ of the enrolled patients within 3-5 months [29]. In this study, the treatment goal was to achieve adequate disease control rather than complete clearance. In a case study involving two patients with GPP, methotrexate was combined with anti-TNF $\alpha$ inhibitors such as infliximab and adalimumab for long-term control of GPP [33]. In this report, the Psoriasis Area and Severity Index (PASI), a disease measure established for plaque psoriasis, was used to assess treatment success in patients with GPP; however, it is worth noting that defined quantitative disease measures were not used. It is well known that methotrexate has an unacceptable safety profile in some patients, including liver toxicity that may lead to treatment discontinuation or limited use. Other adverse events associated with methotrexate include abnormal liver function, gastrointestinal symptoms, and increased risk of infection [29].

\subsubsection{Calcineurin Inhibitors}

Cyclosporine is commonly used to treat patients with GPP [28]. It acts as an immunosuppressive agent through the inhibition of calcineurin phosphatase signaling [30]. In a retrospective study of 102 patients with GPP, cyclosporine treatment resulted in a response in eight patients, but recurrence was common and the clinical disease measures were not defined [6]. Cyclosporine is associated with adverse events such as hypertension, nephrotoxicity, and increased risk of infection, limiting its long-term use for the maintenance treatment of GPP [6].

\subsubsection{Retinoids}

Retinoids are non-immunosuppressive drugs used for the treatment of psoriasis [34]; however, the evidence that supports the use of retinoids for the treatment of patients with GPP is limited. In a retrospective study that included 15 patients with pustular psoriasis, including 10 patients with GPP, acitretin resulted in a good response (defined as 60-90\% clearance of skin lesions) based on prevention of new pustule formation within 3 days of treatment, and most 


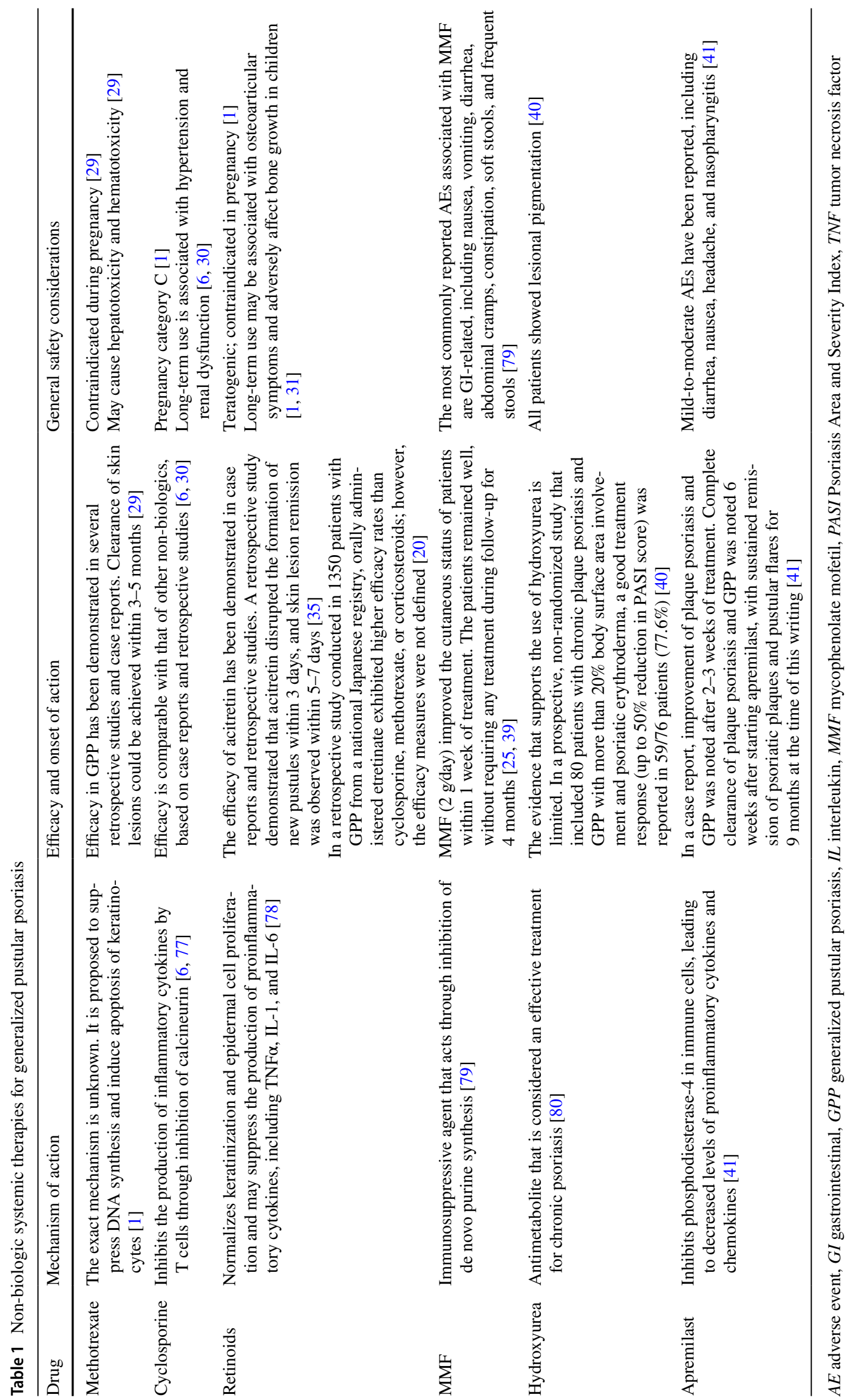


skin lesions resolved within 4-6 weeks [35]. However, it is important to note that relapse can be observed upon acitretin withdrawal [35].

All systemic retinoids are teratogenic [31] and may cause hepatotoxicity [36], mucocutaneous and skeletal toxicities, and hyperlipidemia [37]. Due to the potential toxicity of acitretin and the possibility of disease recurrence following its withdrawal, several study groups have attempted to identify reliable response biomarkers to predict benefit and recurrence following acitretin treatment. A recent study demonstrated that acitretin treatment resulted in a rise in plasma retinol and a reduction in PASI score, suggesting the potential use of plasma retinol as a biomarker of response to acitretin. Additional studies are needed to validate these findings [38].

\subsubsection{Other Non-Biologic Agents}

Several other non-biologic immunomodulatory agents have been used for the treatment of GPP. The evidence that supports their benefit is mainly based on case reports and non-randomized studies. Mycophenolate mofetil has demonstrated efficacy in a case study in which it improved the cutaneous status of a patient with GPP within 1 week of treatment. The patient remained in remission with no use of additional therapies for up to 4 months [39]. Hydroxyurea has also been used despite limited evidence of efficacy. In a prospective, non-randomized study that included 80 patients with chronic plaque psoriasis and GPP with $>20 \%$ body surface area involvement and psoriatic erythroderma, a good treatment response (up to 50\% reduction in PASI score) was reported in 59/76 patients (77.6\%) [40]. Apremilast, a phosphodiesterase-4 inhibitor approved for the treatment of moderate-to-severe plaque psoriasis, has also been used in a patient with GPP and resulted in improvement in GPP symptoms within 2-3 weeks of treatment, and complete skin clearance by Week 6, with sustained remission of psoriatic plaques and pustules for up to 9 months [41]; however, the evidence for its activity is based on a limited number of patients [42]. The positive use of colchicine for the treatment of patients with GPP has also been reported in two case studies [20, 43]; however, no clinical studies have been conducted to investigate the use of colchicine.

In certain cases, topical treatments such as calcipotriene and tacrolimus have been combined with systemic therapies to treat severe disease or used as monotherapy in patients with localized disease [28]. Topical treatments are not recommended for GPP flares; however, they have been proposed in Japanese guidelines for use as maintenance therapy after flares or as adjuvant therapy to manage psoriasis-like symptoms [20]. Alternative treatment modalities have also been used, including triamcinolone with wet body wraps and photochemotherapy using psoralen plus ultraviolet light [28]. In addition, granulocyte/monocyte adsorption apheresis has been used in Japan; however, evidence of its efficacy is mainly based on case reports [20].

\subsection{Biologic Systemic Therapies}

Increased understanding of the inflammatory and autoinflammatory mechanisms involved in the pathogenesis of GPP has provided the rationale for use of targeted biologics to treat GPP (Table 2) [7, 19, 21-23, 26, 33, 34, 44-61].

\subsubsection{TNFa Blocking Agents}

TNF $\alpha$ is a proinflammatory cytokine that plays a central role in the regulation and amplification of inflammatory pathways [62]. The TNF $\alpha$ inhibitors adalimumab, infliximab, and certolizumab pegol are currently approved in Japan for the treatment of GPP [20]. Several case reports have demonstrated the efficacy of TNF $\alpha$ inhibitors in the management of patients with GPP [63-65]. In a retrospective study that involved four patients experiencing GPP flares, treatment with $5 \mathrm{mg} / \mathrm{kg}$ infliximab intravenous infusion at Weeks 0,2 , and 6 , followed by a monthly regimen, was effective. During the first $24 \mathrm{~h}$ after the infliximab infusion, the patients' condition stabilized, as evidenced by the resolution of pustules and suppression of eruption of new pustules within 24-48 h of infliximab infusion [66]. In an open-label study of infliximab that included 10 patients with GPP flares, the time to pustular clearance ranged 1-8 days [55].

Adalimumab was found to be effective and well tolerated for up to 52 weeks for the treatment of 10 Japanese patients with GPP, including those who did not respond to prior treatment with infliximab [67]. In another study that included three patients with GPP treated with adalimumab, time to remission was between 7 and 28 days [55]. Adalimumab has also shown efficacy in combination with acitretin and methotrexate in a case study of two patients [33]. In addition, case reports showed that etanercept was effective in patients with GPP [56], and a case series that included six patients with GPP showed that etanercept treatment normalized laboratory findings and markers of systemic inflammation and reduced PASI scores [68]. Paradoxically, cases of induction of pustular psoriasis flares by TNF $\alpha$ inhibitors have been reported; however, the mechanisms remain to be fully elucidated [7].

\subsubsection{IL-17A and IL-17 Receptor Inhibitors}

IL-17 is a key cytokine produced by T-helper 17 cells and plays an important role in the pathogenesis of inflammatory skin diseases. Moreover, IL-17A acts as a potent inducer of neutrophil recruitment [1]. Brodalumab, an IL-17 receptor antagonist approved in Taiwan and Thailand for 


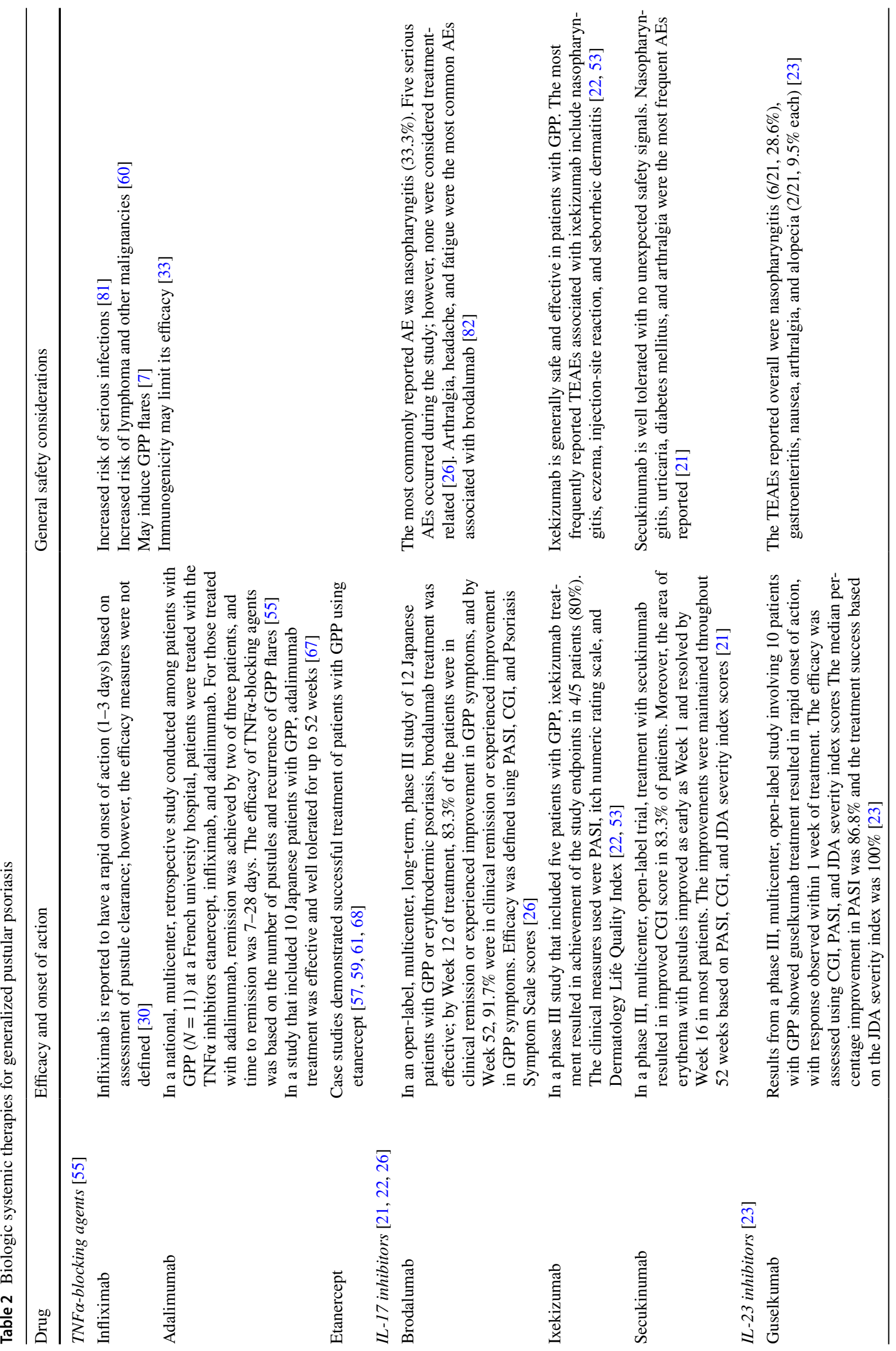




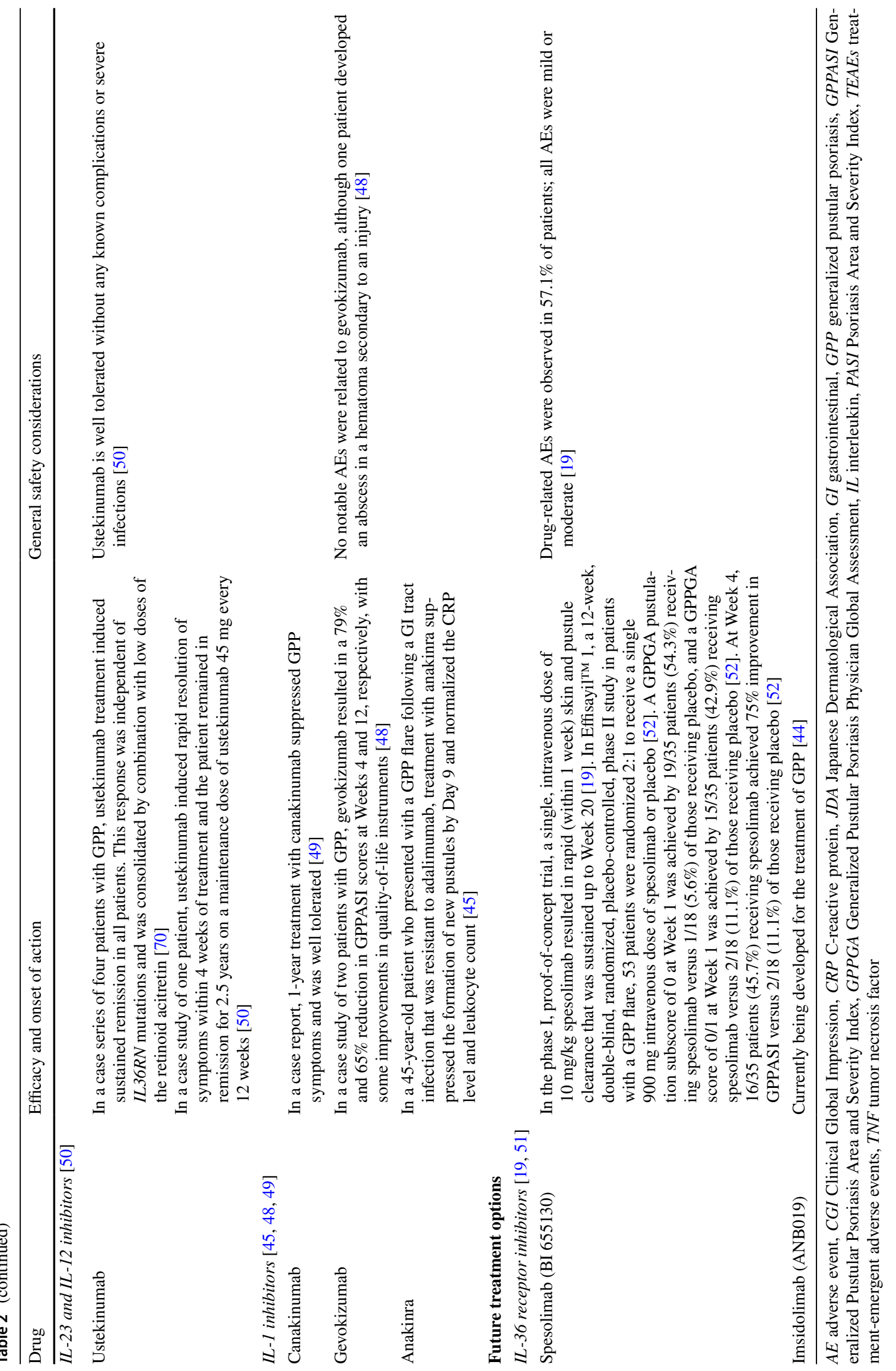


the treatment of GPP, has demonstrated efficacy in small clinical trials [26]. In an open-label, multicenter, long-term, phase III study of 12 Japanese patients with GPP or erythrodermic psoriasis, $83.3 \%$ of the patients treated with brodalumab achieved clinical remission or improved clinical status by Week 12 of treatment. By Week 52, 91.7\% were in clinical remission or had improved clinical status. Similar trends were also observed in the PASI and Psoriasis Symptom Scale scores [26].

Secukinumab, a monoclonal antibody that targets IL$17 \mathrm{~A}$, has demonstrated efficacy in a phase III study of 12 patients with GPP in Japan. Secukinumab monotherapy and co-therapy resulted in $9 / 12$ patients $(75 \%)$ achieving a Clinical Global Impression (CGI) score of 'very much improved' at Week 12 , and $7 / 12$ patients $(58.3 \%)$ achieving this at Week 52 [21]. PASI 75 was achieved by $8 / 12$ patients $(72.7 \%)$ at approximately Weeks $3-4$, which reached the maximum at approximately Week $16(10 / 12$ patients [83.3\%]) and was sustained until Week 52 [21].

Ixekizumab, an IL-17A antagonist, has demonstrated efficacy in patients with GPP in three phase III, open-label, multicenter studies, which included Japanese patients with GPP as a subset [22]. Of the five patients with GPP who were included in the study, four achieved a PASI 75 response, and two had completely clear skin by Week 12 ; the response was maintained for the 52-week treatment period [22]. In the 3-year long-term follow-up study, all five patients with GPP had a Global Improvement Score of resolved or improved from Week 12 onwards. In addition, all patients experienced improvement in PASI scores [69]. The mean PASI score was 12.8 at baseline and 1.8 and 1.6 at Weeks 52 and 244, respectively [69].

\subsubsection{IL-23 and IL-23/IL-12 Inhibitors}

Guselkumab, a monoclonal IL-23 inhibitor, was found to be effective in patients with GPP. In a phase III, multicenter, open-label study in Japan involving 10 patients with GPP, treatment success (defined as a CGI score of 'very much improved', 'much improved', or 'minimally improved') was observed with guselkumab within 1 week in five patients (50\%) with GPP [23]. In this study, guselkumab $50 \mathrm{mg}$ was administrated subcutaneously at Weeks 0 and 4, and every 8 weeks thereafter until Week 52. At Week 52, treatment success was achieved by all eight patients who completed the study [23].

Risankizumab, which targets the p19 subunit of IL-23, is another monoclonal antibody that is approved in Japan for the treatment of patients with GPP [56]. In addition, ustekinumab, a monoclonal antibody targeted against IL-23 and $\mathrm{IL}-12$, has been reported to be effective in a case series of four patients with treatment-refractory GPP regardless of
IL36RN mutation status [70], but no specific disease measures were mentioned.

\subsubsection{IL-1 $\beta$ and IL-1R Inhibitors}

Canakinumab, a monoclonal antibody that targets IL-1 $\beta$, has shown efficacy in a patient who experienced hypersensitivity to the IL-1R antagonist anakinra. Canakinumab treatment resulted in relief of the patient's symptoms based on complete skin clearance and prevention of future recurrence of systemic symptoms [49]. In addition, absolute eosinophil count and liver tests were normalized [49]. Gevokizumab, another monoclonal antibody that blocks the activation of IL-1 $\beta$ receptors, has shown encouraging results in an openlabel, expanded-access study in two patients with severe, recalcitrant GPP. Two patients treated with gevokizumab had a respective $79 \%$ and $65 \%$ reduction in Generalized Pustular Psoriasis Area and Severity Index scores at Week 4 and 12, with some improvements in quality-of-life instruments [48]. Anakinra is an IL-1R antagonist that has the potential to be an effective treatment for GPP. In a case report of a 45-yearold patient who presented with a GPP flare following a gastrointestinal tract infection that was resistant to adalimumab, treatment with anakinra suppressed the formation of new pustules by Day 9 and normalized the C-reactive protein level and leukocyte count [45]. Although these reports demonstrate encouraging results, large, prospective, randomized clinical trials in patients with GPP are needed to confirm the broad efficacy and safety of IL-1-targeted therapies for the treatment of GPP flares.

\section{Future Treatment Options}

\subsection{IL-36 Pathway Inhibitors}

Understanding the central role of the IL-36 pathway in the pathogenesis of GPP has paved the way for the development of novel targeted anti-IL-36 therapies for the treatment of patients with the disease. Spesolimab (BI 655130), a selective, humanized antibody against the IL-36 receptor that blocks its activation and suppresses downstream proinflammatory signaling, has demonstrated efficacy in a phase I, proof-of-concept trial. A single intravenous dose of spesolimab $10 \mathrm{mg} / \mathrm{kg}$ was associated with rapid (within 1 week) clearance of skin and pustules up to Week 20. In seven patients presenting with a GPP flare, spesolimab was associated with rapid and sustained improvements in clinical symptoms. A Generalized Pustular Psoriasis Physician Global Assessment (GPPGA) score of 0 or 1 was achieved within 1 week of treatment by five patients $(71.4 \%)$ and maintained up to Week 20 . Rapid improvements in the signs and symptoms of GPP 


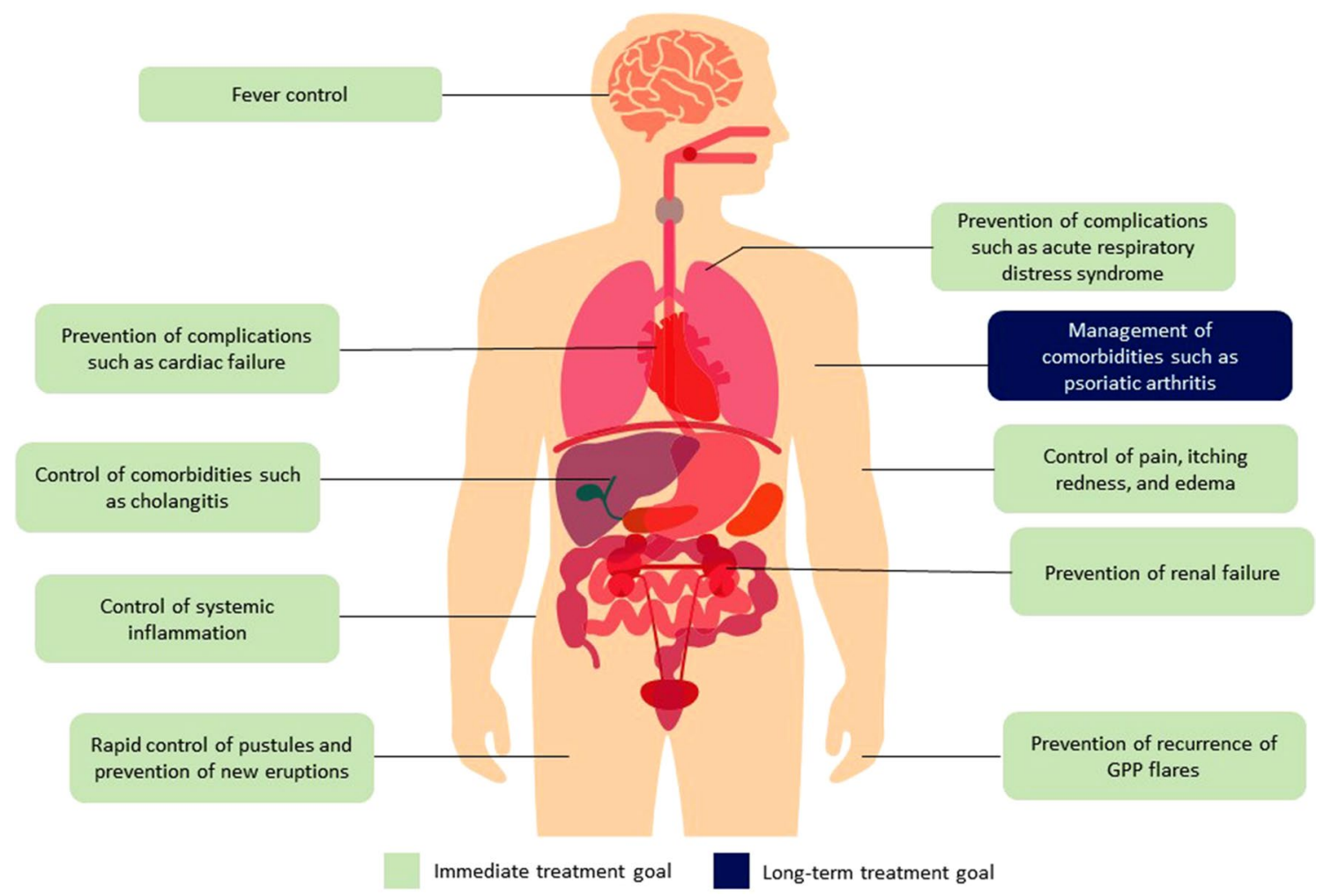

Fig. 2 Treatment goals in patients with GPP. GPP is a systemic disease with manifestations that affect several organs. Treatment goals should focus on improving skin-related symptoms and systemic inflammation. GPP generalized pustular psoriasis

after treatment with spesolimab were achieved according to patient-reported outcomes [19]. Spesolimab was effective regardless of $I L 36 R N$ mutation status, which indicates that the IL-36 pathway is critical to the pathogenesis of GPP irrespective of a patient's genetic background [19].

These findings were confirmed by the results of the Effisayil ${ }^{\mathrm{TM}} 1$ study, a 12-week, double-blind, randomized, placebo-controlled, phase II study in patients with a GPP flare. A total of 53 patients were randomized 2:1 to receive a single $900 \mathrm{mg}$ intravenous dose of spesolimab or placebo [52]. A GPPGA pustulation subscore of 0 at Week 1 was achieved by $19 / 35$ patients $(54.3 \%)$ receiving spesolimab versus $1 / 18(5.6 \%)$ of those receiving placebo (one-sided $p=0.0004$ ) [52]. These results were sustained throughout the 12-week study. Spesolimab had a tolerable safety profile, and most adverse events were mild to moderate and comparable to those in the placebo arm [52]. Spesolimab is currently being evaluated for the prevention of GPP flares in Effisayil ${ }^{\mathrm{TM}}$ 2, a phase IIb, dose-finding study [71].

Several other anti-IL-36 therapies are currently under development. Imsidolimab (ANB019) is a monoclonal antibody targeted against the IL-36 receptor that is currently being developed for several skin indications, including GPP. Positive results of a single-arm, openlabel, phase II trial of imsidolimab in eight patients with
GPP were recently reported [72]. Based on these data, a phase III trial, GEMINI-1, will be conducted, in which 45 patients with GPP flares will be enrolled to receive a single dose of $750 \mathrm{mg}$ intravenous imsidolimab, $300 \mathrm{mg}$ intravenous imsidolimab, or placebo. The primary endpoint of this trial is the proportion of patients achieving clear or almost clear skin as determined by a GPPGA score of 0 or 1 at Week 4 [51]. Patients will subsequently be enrolled in the GEMINI-2 trial to receive monthly doses of 200 $\mathrm{mg}$ subcutaneous imsidolimab or placebo based on their response to treatment in the GEMINI-1 trial [51]. Simi-

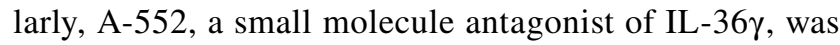
recently identified using high-throughput screening and is being evaluated for the treatment of plaque psoriasis and potentially other inflammatory skin diseases [73].

\section{Establishing Treatment Goals in GPP}

Treatment goals in GPP are not well defined due to the rarity of the disease, its heterogeneous symptoms, and the lack of consistent treatment guidelines and therapeutic monitoring strategies. Clinically relevant treatment goals for GPP flares involve the cessation of pustulation and the resolution of erythema and edema. Several treatment goals can be proposed 
based on the recent advances in our understanding of disease pathogenesis and the development of novel, effective biologics (Fig. 2). Proposed treatment goals can be divided into immediate and long term.

\subsection{Immediate Treatment Goals}

The main immediate therapeutic goals during a GPP flare are to improve skin symptoms and reduce the burden of systemic manifestations to prevent potential complications [56]. Rapid control of skin symptoms, within a week of treatment, is a feasible treatment goal based on the results of spesolimab clinical trials [19]. Short-term treatment goals should also focus on the prevention of further complications such as neutrophilic cholangitis, uveitis, acute respiratory distress syndrome, cardiovascular aseptic shock, heart failure, prerenal kidney failure, and severe infections.

\subsection{Long-Term Treatment Goals}

In the long-term management of GPP, treatment goals should focus on the prevention of new flares or disease worsening and the treatment of GPP comorbidities such as hypertension, diabetes mellitus, hyperlipidemia, ischemic heart disease, osteoarthritis, and cholangitis, with the ultimate goal of improving patients' quality of life [15, 74]. Specific long-term treatment goals are not well defined in clinical trials; however, the results of a recent survey of Corrona Registry dermatologists indicated that in $>80 \%$ of patients, symptoms still persist in between flares, and many treatments are not effective at preventing flares [75]. These findings highlight the need for rigorous real-world studies to further evaluate current and emerging GPP treatment options and define feasible long-term goals to improve patients' quality of life.

\subsection{Other Treatment Goals}

During pregnancy, GPP flares should be treated promptly to prevent any further complications that may impact the well-being of the mother and fetus [76]. In addition, treatment goals of infantile and juvenile pustular psoriasis should focus on preventing recurrences, which have been reported to occur annually [3]. Furthermore, treatment of geriatric patients with GPP may pose substantial challenges due to comorbidities and potential exposure to medications that could trigger flares.

The identification of patient-desired treatment goals is needed to improve patients' quality of life and alleviate the emotional burden caused by the psychological or physical pain associated with a GPP diagnosis (these are discussed in greater detail in Chapter 7 [https://doi.org/10.1007/s40257021-00663-y] of this supplement).

\section{Conclusions}

International treatment guidelines for patients with GPP are lacking and most available treatments are used off-label based on efficacy in plaque psoriasis or limited evidence derived from case reports and small, uncontrolled, openlabel, single-arm trials in GPP. Recent advances in our understanding and treatment of GPP offer several opportunities to improve patient care. Moreover, there is a lack of consensus regarding the definition of treatment success or failure and the optimal time to switch patients to an alternative treatment. With the emergence of new and effective therapeutic interventions, patient care will continue to evolve towards improving therapeutic outcomes and quality of life.

Acknowledgements All authors meet the criteria for authorship as recommended by the International Committee of Medical Journal Editors (ICMJE) and made the decision to submit the manuscript for publication. The authors did not receive payment related to the development of the manuscript. Agreements between Boehringer Ingelheim and the authors included the confidentiality of the study data. Yasser Heakal, $\mathrm{PhD}$, of OPEN Health Communications (London, UK) provided medical writing, editorial and/or formatting support, which was contracted and funded by Boehringer Ingelheim. Boehringer Ingelheim was given the opportunity to review the manuscript for medical and scientific accuracy as well as intellectual property considerations.

\section{Declarations}

Disclosure statement This article has been published as part of a journal supplement wholly funded by Boehringer Ingelheim.

Funding Medical writing support was funded by Boehringer Ingelheim.

Conflicts of interest James Krueger has received grants from and been an investigator for Boehringer Ingelheim; received personal fees from AbbVie, Baxter, Biogen Idec, Delenex Therapeutics, Kineta, Sanofi, Serono, and XenoPort; and received grants from Amgen, Bristol Myers Squibb, Dermira, Innovaderm Research, Janssen, Kadmon, Kyowa Kirin, Eli Lilly, Merck, Novartis, Parexel, and Pfizer. Lluís Puig has received grants/research support or participated in clinical trials (paid to institution) from AbbVie, Almirall, Amgen, Boehringer Ingelheim, Celgene, Janssen, LEO Pharma, Lilly, Novartis, Pfizer, Regeneron, Roche, Sanofi, and UCB; received honoraria or consultation fees from AbbVie, Almirall, Amgen, Baxalta, Biogen, Boehringer Ingelheim, Bristol Myers Squibb, Celgene, Fresenius-Kabi, Janssen, JS BIOCAD, LEO Pharma, Lilly, Mylan, Novartis, Pfizer, Regeneron, Roche, Sandoz, Samsung-Bioepis, Sanofi, and UCB; and participated in company-sponsored speaker's bureau for Celgene, Janssen, Lilly, Novartis, and Pfizer. Diamant Thaçi has served as a consultant, advisory board member, and/or investigator for Pfizer, AbbVie, Almirall, Amgen, Beiersdorf, Bristol Myers Squibb, Boehringer Ingelheim, Dainippon Sumitomo Pharma, Eli Lilly, Galapagos, GlaxoSmithKline, JanssenCilag, LEO Pharma, Maruho, Medac, MorphoSys, Novartis, Regeneron, Samsung, Sandoz, Sanofi, Sun Pharma, and UCB.

Availability of data and material Not applicable.

Code availability Not applicable. 
Author contributions The sponsor of the supplement and all authors identified and discussed the concept for each chapter and the supplement as a whole; further development of each review article was at the direction of the authors, who provided guidance to OPEN Health Communications on the content of the article, critically revised the work, and approved the content for publication.

Ethics approval Not applicable.

Consent to participate Not applicable.

Consent for publication Not applicable.

Open Access This article is licensed under a Creative Commons Attribution-NonCommercial 4.0 International License, which permits any non-commercial use, sharing, adaptation, distribution and reproduction in any medium or format, as long as you give appropriate credit to the original author(s) and the source, provide a link to the Creative Commons licence, and indicate if changes were made. The images or other third party material in this article are included in the article's Creative Commons licence, unless indicated otherwise in a credit line to the material. If material is not included in the article's Creative Commons licence and your intended use is not permitted by statutory regulation or exceeds the permitted use, you will need to obtain permission directly from the copyright holder. To view a copy of this licence, visit http://creativecommons.org/licenses/by-nc/4.0/.

\section{References}

1. Gooderham MJ, Van Voorhees AS, Lebwohl MG. An update on generalized pustular psoriasis. Expert Rev Clin Immunol. 2019;15(9):907-19.

2. Navarini AA, Burden AD, Capon F, Mrowietz U, Puig L, Koks $\mathrm{S}$, et al. European consensus statement on phenotypes of pustular psoriasis. J Eur Acad Dermatol Venereol. 2017;31(11):1792-9.

3. Benjegerdes KE, Hyde K, Kivelevitch D, Mansouri B. Pustular psoriasis: pathophysiology and current treatment perspectives. Psoriasis (Auckl). 2016;6:131-44.

4. Zelickson BD, Muller SA. Generalized pustular psoriasis. A review of 63 cases. Arch Dermatol. 1991;127(9):1339-45.

5. Takedai T, Yamamoto I, Tokeshi J. Acute generalized pustular psoriasis presenting with erythroderma associated with shock and acute renal failure. Hawaii Med J. 2003;62(12):278-81.

6. Choon SE, Lai NM, Mohammad NA, Nanu NM, Tey KE, Chew SF. Clinical profile, morbidity, and outcome of adult-onset generalized pustular psoriasis: analysis of 102 cases seen in a tertiary hospital in Johor, Malaysia. Int J Dermatol. 2014;53(6):676-84.

7. Brunasso AM, Laimer M, Massone C. Paradoxical reactions to targeted biological treatments: a way to treat and trigger? Acta Derm Venereol. 2010;90(2):183-5.

8. Baker H, Ryan TJ. Generalized pustular psoriasis. A clinical and epidemiological study of 104 cases. Br J Dermatol. 1968;80(12):771-93.

9. Marrakchi S, Guigue P, Renshaw BR, Puel A, Pei XY, Fraitag S, et al. Interleukin-36-receptor antagonist deficiency and generalized pustular psoriasis. N Engl J Med. 2011;365(7):620-8.

10. Onoufriadis A, Simpson MA, Pink AE, Di Meglio P, Smith CH, Pullabhatla V, et al. Mutations in IL36RN/IL1F5 are associated with the severe episodic inflammatory skin disease known as generalized pustular psoriasis. Am J Hum Genet. 2011;89(3):432-7.

11. Farooq M, Nakai H, Fujimoto A, Fujikawa H, Matsuyama A, Kariya N, et al. Mutation analysis of the IL36RN gene in 14
Japanese patients with generalized pustular psoriasis. Hum Mutat. 2013;34(1):176-83.

12. Sugiura K, Takemoto A, Yamaguchi M, Takahashi H, Shoda Y, Mitsuma T, et al. The majority of generalized pustular psoriasis without psoriasis vulgaris is caused by deficiency of interleukin-36 receptor antagonist. J Invest Dermatol. 2013;133(11):2514-21.

13. Wang TS, Chiu HY, Hong JB, Chan CC, Lin SJ, Tsai TF. Correlation of IL36RN mutation with different clinical features of pustular psoriasis in Chinese patients. Arch Dermatol Res. 2016;308(1):55-63.

14. Tauber M, Bal E, Pei XY, Madrange M, Khelil A, Sahel H, et al. IL36RN mutations affect protein expression and function: a basis for genotype-phenotype correlation in pustular diseases. J Invest Dermatol. 2016;136(9):1811-9.

15. Bachelez H. Pustular psoriasis: the dawn of a new era. Acta Derm Venereol. 2020;100(3):adv00034.

16. Arakawa A, Vollmer S, Besgen P, Galinski A, Summer B, Kawakami Y, et al. Unopposed IL-36 activity promotes clonal CD4(+) T-cell responses with IL-17A production in generalized pustular psoriasis. J Invest Dermatol. 2018;138(6):1338-47.

17. Buhl AL, Wenzel J. Interleukin-36 in infectious and inflammatory skin diseases. Front Immunol. 2019;10:1162.

18. Zhou J, Luo Q, Cheng Y, Wen X, Liu J. An update on genetic basis of generalized pustular psoriasis (review). Int J Mol Med. 2021;47(6): 118

19. Bachelez H, Choon SE, Marrakchi S, Burden AD, Tsai TF, Morita A, et al. Inhibition of the interleukin-36 pathway for the treatment of generalized pustular psoriasis. N Engl J Med. 2019;380(10):981-3.

20. Fujita H, Terui T, Hayama K, Akiyama M, Ikeda S, Mabuchi T, et al. Japanese guidelines for the management and treatment of generalized pustular psoriasis: the new pathogenesis and treatment of GPP. J Dermatol. 2018;45(11):1235-70.

21. Imafuku S, Honma M, Okubo Y, Komine M, Ohtsuki M, Morita A, et al. Efficacy and safety of secukinumab in patients with generalized pustular psoriasis: a 52-week analysis from phase III openlabel multicenter Japanese study. J Dermatol. 2016;43(9):1011-7.

22. Saeki H, Nakagawa H, Nakajo K, Ishii T, Morisaki Y, Aoki T, et al. Efficacy and safety of ixekizumab treatment for Japanese patients with moderate to severe plaque psoriasis, erythrodermic psoriasis and generalized pustular psoriasis: results from a 52-week, open-label, phase 3 study (UNCOVER-J). J Dermatol. 2017;44(4):355-62.

23. Sano S, Kubo H, Morishima H, Goto R, Zheng R, Nakagawa H. Guselkumab, a human interleukin-23 monoclonal antibody in Japanese patients with generalized pustular psoriasis and erythrodermic psoriasis: efficacy and safety analyses of a 52-week, phase 3, multicenter, open-label study. J Dermatol. 2018;45(5):529-39.

24. UCB. CIMZIA ${ }^{\circledR}$ (certolizumab pegol) now available for patients in Japan living with multiple psoriatic diseases. Available at: https://www.ucb.com/stories-media/Press-Releases/article/CIMZIA-certolizumab-pegol-now-Available-for-Patients-in-Japan-living-with-Multiple-Psoriatic-Diseases. Accessed Aug 2021.

25. Wilsmann-Theis D, Schnell LM, Ralser-Isselstein V, Bieber T, Schon MP, Huffmeier U, et al. Successful treatment with interleukin-17A antagonists of generalized pustular psoriasis in patients without IL36RN mutations. J Dermatol. 2018;45(7):850-4.

26. Yamasaki K, Nakagawa H, Kubo Y, Ootaki K, Japanese Brodalumab Study G. Efficacy and safety of brodalumab in patients with generalized pustular psoriasis and psoriatic erythroderma: results from a 52-week, open-label study. Br J Dermatol. 2017;176(3):741-51.

27. Pharmaceuticals and Medical Devices Agency, Japan. Report on the deliberation of results. Tremfya subcutaneous injection 100 mg syringe (guselkumab). Available at: https://www.pmda.go.jp/ files/000234741.pdf. Accessed Aug 2021. 
28. Robinson A, Van Voorhees AS, Hsu S, Korman NJ, Lebwohl MG, Bebo BF Jr, et al. Treatment of pustular psoriasis: from the Medical Board of the National Psoriasis Foundation. J Am Acad Dermatol. 2012;67(2):279-88.

29. Haustein UF, Rytter M. Methotrexate in psoriasis: 26 years' experience with low-dose long-term treatment. J Eur Acad Dermatol Venereol. 2000;14(5):382-8.

30. Zhou LL, Georgakopoulos JR, Ighani A, Yeung J. Systemic monotherapy treatments for generalized pustular psoriasis: a systematic review. J Cutan Med Surg. 2018;22(6):591-601.

31. David M, Hodak E, Lowe NJ. Adverse effects of retinoids. Med Toxicol Adverse Drug Exp. 1988;3(4):273-88.

32. Yan K, Xu W, Huang Y, Zhang Z, Huang Q, Xin KZ, et al. Methotrexate restores the function of peripheral blood regulatory $\mathrm{T}$ cells in psoriasis vulgaris via the CD73/AMPK/mTOR pathway. Br J Dermatol. 2018;179(4):896-905.

33. Kawakami H, Maeda T, Abe N, Matsumoto Y, Mitsuhashi Y, Tsuboi R, et al. Efficacy of adalimumab and methotrexate combination therapy on generalized pustular psoriasis patients unresponsive to infliximab monotherapy due to anti-infliximab antibody development. J Dermatol. 2015;42(1):94-5.

34. Kang S, Li XY, Voorhees JJ. Pharmacology and molecular action of retinoids and vitamin D in skin. J Investig Dermatol Symp Proc. 1996;1(1):15-21.

35. Chen P, Li C, Xue R, Chen H, Tian X, Zeng K, et al. Efficacy and safety of acitretin monotherapy in children with pustular psoriasis: results from 15 cases and a literature review. J Dermatol Treat. 2018;29(4):353-63.

36. Otley CC, Stasko T, Tope WD, Lebwohl M. Chemoprevention of nonmelanoma skin cancer with systemic retinoids: practical dosing and management of adverse effects. Dermatol Surg. 2006;32(4):562-8.

37. Lee CS, Li K. A review of acitretin for the treatment of psoriasis. Expert Opin Drug Saf. 2009;8(6):769-79.

38. Yang H, Tan Q, Chen GH, Chen JS, Fu Z, Ren FL, et al. Plasma retinol as a predictive biomarker of disease activity and response to acitretin monotherapy in children with generalized pustular psoriasis. J Eur Acad Dermatol Venereol. 2020;34(6):e270-2.

39. Ji YZ, Geng L, Ma XH, Wu Y, Zhou HB, Li B, et al. Severe generalized pustular psoriasis treated with mycophenolate mofetil. J Dermatol. 2011;38(6):603-5.

40. Das S, Das S. Hydroxyurea-an experience in the management of psoriasis. J Pak Assoc Dermatol. 2013;23(4):401-6.

41. Jeon C, Nakamura M, Sekhon S, Yan D, Wu JJ, Liao W, et al. Generalized pustular psoriasis treated with apremilast in a patient with multiple medical comorbidities. JAAD Case Rep. 2017;3(6):495-7.

42. Kromer C, Loewe E, Schaarschmidt ML, Pinter A, Gerdes S, Herr R, et al. Drug survival in the treatment of generalized pustular psoriasis: a retrospective multicenter study. Dermatol Ther. 2021;34(2):e14814.

43. Zachariae H, Kragballe K, Herlin T. Colchicine in generalized pustular psoriasis: clinical response and antibody-dependent cytotoxicity by monocytes and neutrophils. Arch Dermatol Res. 1982;274(3-4):327-33.

44. ClinicalTrials.gov. A study to evaluate the efficacy and safety of ANB019 in subjects with generalized pustular psoriasis (GPP). Available at: https://clinicaltrials.gov/ct2/show/NCT03619902. Accessed 11 Aug 2021.

45. Huffmeier U, Watzold M, Mohr J, Schon MP, Mossner R. Successful therapy with anakinra in a patient with generalized pustular psoriasis carrying IL36RN mutations. Br J Dermatol. 2014;170(1):202-4.

46. Husson B, Barbe C, Hegazy S, Seneschal J, Aubin F, Mahe E, et al. Efficacy and safety of TNF blockers and of ustekinumab in palmoplantar pustulosis and in acrodermatitis continua of Hallopeau. J Eur Acad Dermatol Venereol. 2020;34(10):2330-8.

47. Johnston A, Xing X, Wolterink L, Barnes DH, Yin Z, Reingold L, et al. IL-1 and IL-36 are dominant cytokines in generalized pustular psoriasis. J Allergy Clin Immunol. 2017;140(1):109-20.

48. Mansouri B, Richards L, Menter A. Treatment of two patients with generalized pustular psoriasis with the interleukin-1beta inhibitor gevokizumab. Br J Dermatol. 2015;173(1):239-41.

49. Skendros P, Papagoras C, Lefaki I, Giatromanolaki A, Kotsianidis I, Speletas M, et al. Successful response in a case of severe pustular psoriasis after interleukin-1beta inhibition. Br J Dermatol. 2017;176(1):212-5.

50. Storan ER, O'Gorman SM, Markham T. Generalized pustular psoriasis treated with ustekinumab. Clin Exp Dermatol. 2016;41(6):689-90.

51. AnaptyBio. Imsidolimab. Available at: https://www.anaptysbio. com/pipeline/imsidolimab/. Accessed 11 Aug 2021.

52. Bachelez H CS, Marrakchi S, Burden AD, Tsai TF, Morita A, et al. Effisayil 1: a multicenter, randomized, double-blind, placebo-controlled study to evaluate the efficacy, safety, and tolerability of spesolimab in patients with a generalized pustular psoriasis flare. The World Psoriasis and Psoriatic Arthritis Conference. Stockholm; 30 June-3 July 2021. p. 35129.

53. Saeki H, Nakagawa H, Ishii T, Morisaki Y, Aoki T, Berclaz PY, et al. Efficacy and safety of open-label ixekizumab treatment in Japanese patients with moderate-to-severe plaque psoriasis, erythrodermic psoriasis and generalized pustular psoriasis. J Eur Acad Dermatol Venereol. 2015;29(6):1148-55.

54. Tsai YC, Tsai TF. Anti-interleukin and interleukin therapies for psoriasis: current evidence and clinical usefulness. Ther Adv Musculoskelet Dis. 2017;9(11):277-94.

55. Viguier M, Aubin F, Delaporte E, Pages C, Paul C, Beylot-Barry $M$, et al. Efficacy and safety of tumor necrosis factor inhibitors in acute generalized pustular psoriasis. Arch Dermatol. 2012;148(12):1423-5.

56. Wang WM, Jin HZ. Biologics in the treatment of pustular psoriasis. Expert Opin Drug Saf. 2020;19(8):969-80.

57. Cuperus E, Koevoets R, van der Smagt JJ, Toonstra J, de Graaf $\mathrm{M}$, Frenkel J, et al. Juvenile interleukin-36 receptor antagonist deficiency (DITRA) with c.80T $>$ C (p.Leu27Pro) mutation successfully treated with etanercept and acitretin. JAAD Case Rep. 2018;4(2):192-5.

58. Esposito M, Mazzotta A, Saraceno R, Schipani C, Chimenti S. Influence and variation of the body mass index in patients treated with etanercept for plaque-type psoriasis. Int J Immunopathol Pharmacol. 2009;22(1):219-25.

59. Fialova J, Vojackova N, Vanousova D, Hercogova J. Juvenile generalized pustular psoriasis treated with etanercept. Dermatol Ther. 2014;27(2):105-8.

60. Fiorentino D, Ho V, Lebwohl MG, Leite L, Hopkins L, Galindo $\mathrm{C}$, et al. Risk of malignancy with systemic psoriasis treatment in the Psoriasis Longitudinal Assessment Registry. J Am Acad Dermatol. 2017;77(5):845-54 e5.

61. Kamarashev J, Lor P, Forster A, Heinzerling L, Burg G, Nestle FO. Generalised pustular psoriasis induced by cyclosporin a withdrawal responding to the tumour necrosis factor alpha inhibitor etanercept. Dermatology. 2002;205(2):213-6.

62. Yost J, Gudjonsson JE. The role of TNF inhibitors in psoriasis therapy: new implications for associated comorbidities. F1000 Med Rep. 2009;1:30.

63. Routhouska SB, Sheth PB, Korman NJ. Long-term management of generalized pustular psoriasis with infliximab: case series. J Cutan Med Surg. 2008;12(4):184-8.

64. Poulalhon N, Begon E, Lebbe C, Liote F, Lahfa M, Bengoufa $\mathrm{D}$, et al. A follow-up study in 28 patients treated with infliximab for severe recalcitrant psoriasis: evidence for efficacy and 
high incidence of biological autoimmunity. Br J Dermatol. 2007;156(2):329-36.

65. Adachi A, Komine M, Hirano T, Tsuda H, Karakawa M, Murata $\mathrm{S}$, et al. Case of generalized pustular psoriasis exacerbated during pregnancy, successfully treated with infliximab. J Dermatol. 2016;43(12):1439-40.

66. Trent JT, Kerdel FA. Successful treatment of Von Zumbusch pustular psoriasis with infliximab. J Cutan Med Surg. 2004;8(4):224-8.

67. Morita A, Yamazaki F, Matsuyama T, Takahashi K, Arai S, Asahina A, et al. Adalimumab treatment in Japanese patients with generalized pustular psoriasis: results of an open-label phase 3 study. J Dermatol. 2018;45(12):1371-80.

68. Esposito M, Mazzotta A, Casciello C, Chimenti S. Etanercept at different dosages in the treatment of generalized pustular psoriasis: a case series. Dermatology. 2008;216(4):355-60.

69. Okubo Y, Mabuchi T, Iwatsuki K, Elmaraghy H, Torisu-Itakura $\mathrm{H}$, Morisaki $\mathrm{Y}$, et al. Long-term efficacy and safety of ixekizumab in Japanese patients with erythrodermic or generalized pustular psoriasis: subgroup analyses of an open-label, phase 3 study (UNCOVER-J). J Eur Acad Dermatol Venereol. 2019;33(2):325-32.

70. Arakawa A, Ruzicka T, Prinz JC. Therapeutic efficacy of interleukin 12/interleukin 23 blockade in generalized pustular psoriasis regardless of IL36RN mutation status. JAMA Dermatol. 2016;152(7):825-8.

71. ClinicalTrials.gov. A study to test whether BI 655130 (spesolimab) prevents flare-ups in patients with generalized pustular psoriasis. Available at: https://clinicaltrials.gov/ct2/show/NCT04 399837. Accessed 11 Aug 2021.

72. Gudjonsson J RA, Barker J, Pink A, Reynolds N, Griffiths C, et al. Imsidolimab, an anti-IL-36 receptor monoclonal antibody, in the treatment of generalized pustular psoriasis: results from a phase 2 trial. 30th EADV Congress; 29 September-2 October 2021.

73. Todorovic V, Su Z, Putman CB, Kakavas SJ, Salte KM, McDonald HA, et al. Small molecule IL-36 gamma antagonist as a novel therapeutic approach for plaque psoriasis. Sci Rep. 2019;9(1):9089.

74. Miyachi H, Konishi T, Kumazawa R, Matsui H, Shimizu S, Fushimi K, et al. Treatments and outcomes of generalized pustular psoriasis: a cohort of 1516 patients in a nationwide inpatient database in Japan. J Am Acad Dermatol. 2021. https://doi.org/10.1016/j. jaad.2021.06.008 (Epub 8 Jun 2021).

75. Strober B, Kotowsky N, Medeiros R, Mackey RH, Harrold LR, Valdecantos WC, et al. Unmet medical needs in the treatment and management of generalized pustular psoriasis flares: evidence from a survey of corrona registry dermatologists. Dermatol Ther (Heidelb). 2021;11(2):529-41.

76. Trivedi MK, Vaughn AR, Murase JE. Pustular psoriasis of pregnancy: current perspectives. Int $\mathrm{J}$ Womens Health. 2018;10:109-15.

77. Ho S, Clipstone N, Timmermann L, Northrop J, Graef I, Fiorentino D, et al. The mechanism of action of cyclosporin A and FK506. Clin Immunol Immunopathol. 1996;80(3 Pt 2):S40-5.

78. Umezawa Y, Ozawa A, Kawasima T, Shimizu H, Terui T, Tagami $\mathrm{H}$, et al. Therapeutic guidelines for the treatment of generalized pustular psoriasis (GPP) based on a proposed classification of disease severity. Arch Dermatol Res. 2003;295(Suppl 1):S43-54.

79. Park $\mathrm{H}$. The emergence of mycophenolate mofetilin dermatology: from its roots in the world of organ transplantation to its versatile role in the dermatology treatment room. J Clin Aesthet Dermatol. 2011;4(1):18-27.

80. Lee ES, Heller MM, Kamangar F, Park K, Liao W, Koo J. Hydroxyurea for the treatment of psoriasis including in HIV-infected individuals: a review. Psoriasis Forum. 2011;17(3):180-7.

81. Penso L, Dray-Spira R, Weill A, Pina Vegas L, Zureik M, Sbidian E. Association between biologics use and risk of serious infection in patients with psoriasis. JAMA Dermatol. 2021;157(9):1056-65.

82. Lebwohl M, Strober B, Menter A, Gordon K, Weglowska J, Puig L, et al. Phase 3 studies comparing brodalumab with ustekinumab in psoriasis. N Engl J Med. 2015;373(14):1318-28. 